\title{
Virtual Blowgun System for Breathing Movement Exercise
}

\author{
Peichao Yu, Kazuhito Shiratori, and Jun'ichi Hoshino \\ University of Tsukuba, Graduate school of Systems and Information Engineering, \\ 1-1-1 Tennodai, Tsukuba-shi, Ibaraki, Japan \\ admin@nerocrux.org, c_tori@mbb.nifty.ne.jp, \\ jhoshino@esys.tsukuba.ac.jp
}

\begin{abstract}
Breathing is the most basic requirement for having good health. However, unhealthy breathing like overbreathing and hyperventilation will happened easily without any awareness. We propose an experimental breathing movement exercise system - Virtual Blowgun System (VBS), offering an easy way of breathing exercise, for people of different physical strength, without space and safety limitations.
\end{abstract}

Keywords: breathing movement exercise, view-dependent virtual reality, versatile training field, blowgun interface.

\section{Introduction}

Breathing is the most basic requirement for having good health. However, unhealthy breathing like overbreathing and hyperventilation will happened easily without any awareness. It upsets the basic rhythm of life and the body works less effectively by causing blocked nose, insomnia, snoring, sleep apnea, panic, anxiety and depression. [1] Furthermore, unhealthy breathing may also cause much more serious diseases, such as allergies, asthma, COPD and emphysema.

There are several breathing methods, such as Buteyko or Pranayama [2], proved to be effective in the treatment of diseases in breath, or in breathing enhancement.

In Japan, a more popular breathing method called "abdominal breathing method" is easy to practice, especially through playing a traditional Japanese sports game, sports blowgun. Through playing this game in a proper way, breathing can be well controlled and exercised. [9]

However, sports blowgun requires wide space and strict safety care. Children, elderly people, or people with disabilities can hardly playing this game, and will not able to compete with ordinary mature adults. 
We propose an experimental breathing movement exercise system - Virtual Blowgun System (VBS). The aim of VBS project is to offer an easy way of breathing exercise, for people of different physical strength, without space, safety or other limitations.

VBS will offer a highly simulated physical and visual experiment as playing the real blowgun sports. Users will also enjoy their exercises with game contents designed for breathing exercise.

\section{Relevant Study}

Wijnand's research [3] shows the importance of virtual environment on helping boost motivation for users training at home.

Virku[4] makes it possible for users to control the avatar in the game by using the exercise bicycle. Game contents are used to help keeping motivation of training.

Tsuji's research [5] on sports skill training focuses on the controlling of the movement of users' body parts.

Masuko's research [6] reflects heart rate on exercise game, which provides an easier way of designing applications, through which both user's motivation and exercise efficiency can be proved.

These systems are able to help user training at home, using virtual reality. However, we found out that the interactive is quite simple. Visually, users can hardly acquire immersive feelings, which will attenuate their motivation of training.

There are devices designed for breathing exercise or long-term medical treatment [7], but there are no interaction, no competition when using these devices. Without these elements, almost all users will feel tired and boring and lack of motivation in a couple of hours after using these devices.

Through our research, we found out that it is important and necessary, to enable virtual reality in creating training system, in order to provide interaction and challenge of archieving goals, which provides motivation of exercising.

\section{System Concept and Design}

VBS consists with 3 parts: blowgun device, displays and USB camera. A PC is also required for data processing and 3D scene generation.

We use large-scale design, for using in exhibitions and public facilities, which requires 2 projectors for front projection, and another projector for floor projection. System setup plan is shown on Fig. 1 .

The system can also be used at home or office, by simply connect the blowgun device to the PC through USB interface. With a USB camera connected, users can also enjoy breathing exercises, sitting or standing in front of a personal computer, by playing game contents for breathing exercises. 


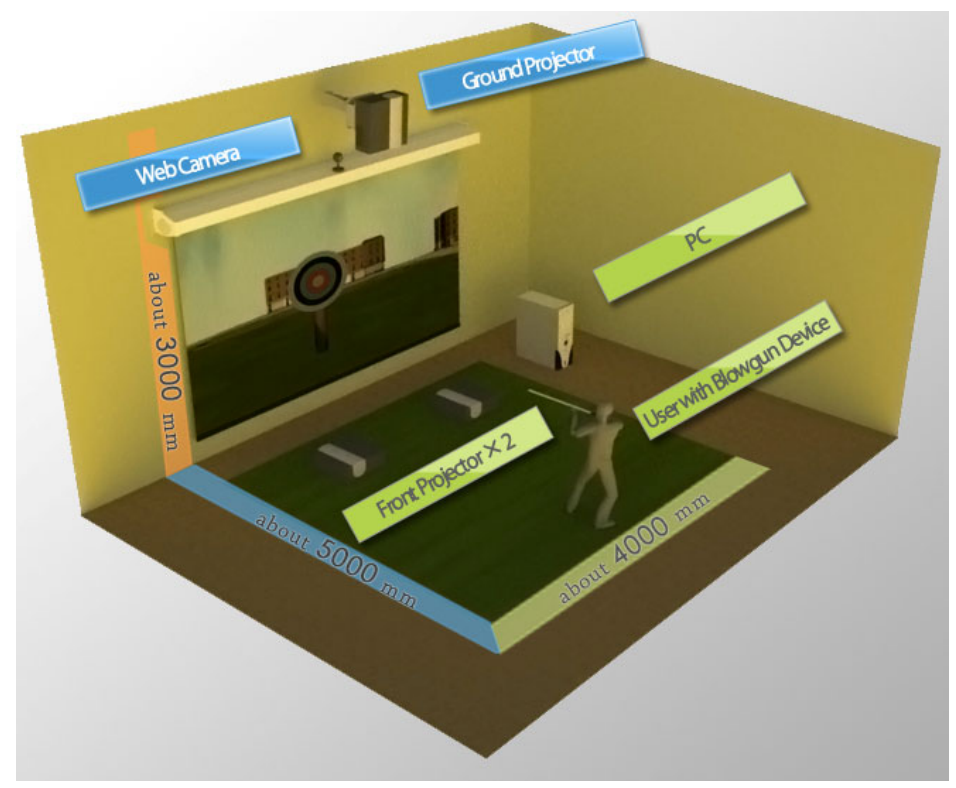

Fig. 1. Floor plan of VBS

Fig. 2 shows the specific structure and dataflow of VBS, which consisted with three parts: virtual blowgun device, USB camera for View-dependent Virtual Reality and PC application.

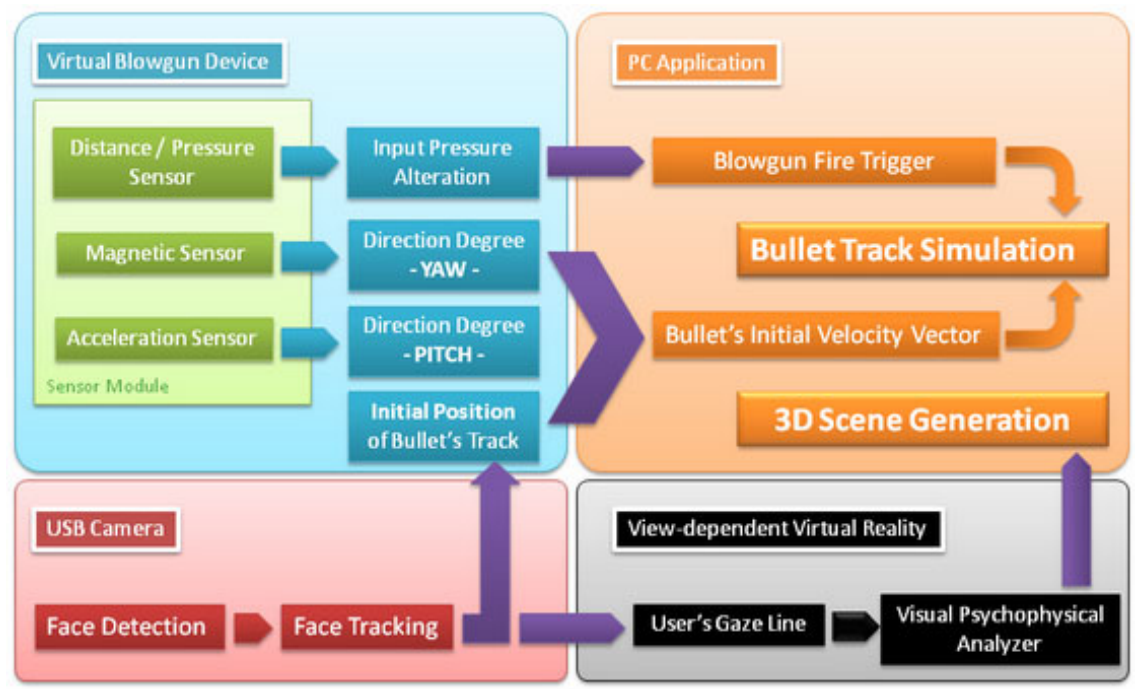

Fig. 2. System structure of VBS 


\subsection{Virtual Blowgun Device}

The pipe-like device is the main input device of our system. We use a aluminum pipe (length of $1200 \mathrm{~mm}$ for training field and exhibition use, $500 \mathrm{~mm}$ for home use, width of $\varphi 15 \mathrm{~mm})$ as the body of the device.

On one side of the pipe, sensor module and pressure controller are installed. And on the other side of the pipe is replaceable mouth piece, through which user blow air into the device.

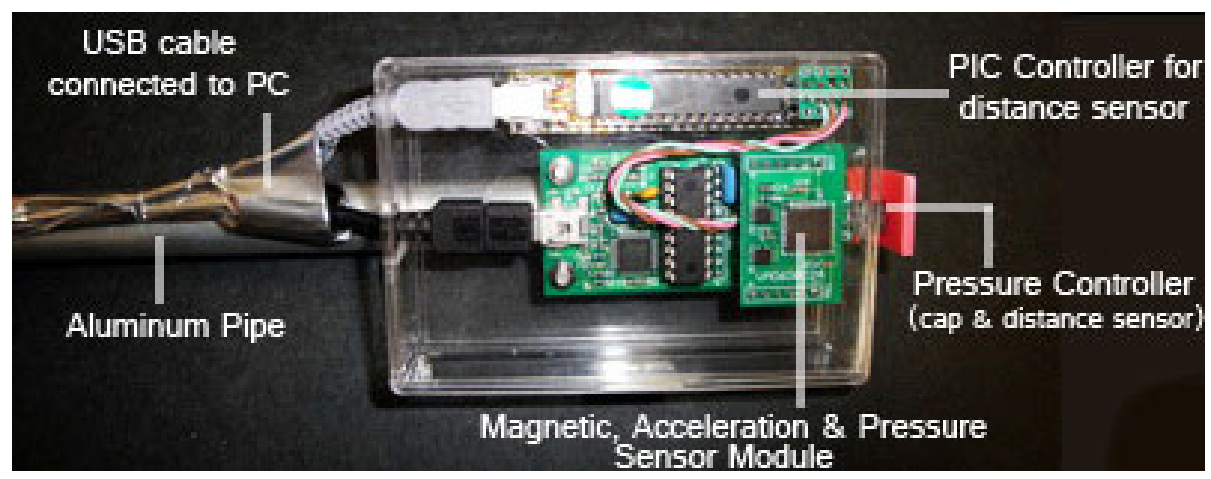

Fig. 3. VBS device structure

Fig. 3 shows the design of the key part: the sensor module and pressure controller of the VBS device. Currently we are still working on the prototype of the device, so all parts of the device have not been combined into one board yet. We have a PIC controller for data processing, and an IR distance sensor, a magnetic sensor, an acceleration sensor and a pressure sensor installed on the pipe.

A pressure controller is also designed, for measuring and controlling of the pressure inside the pipe.

\subsection{USB Camera}

USB Camera is designed to be set upon the center of the display. Manual calibration (inclination angle, position) is required to make sure user's face is in the center of the image camera captures.

The image taken by the camera is used for the generation of view-dependent virtual reality. Details will be explained in Section 4.1.

\subsection{PC Application and Displays}

PC application we created does the following works:

- Processes all the data retrieved from all sensors through serial ports.

- Graphical representation of 3D scene using OpenGL.

- Face detection and tracking using OpenCV.

- Audio effects using OpenAL. 
The virtual scene we built is based on the image of European old town. We suppose the training is on the grass outdoor. The front and ground projectors working seamlessly, offer users a half-surrounded 3 dimensional VR exercise environment.

\subsection{Concept and Rules}

The user is asked to hold the VBS device and try his/her best to aim the center of the target and blow into the device.

When the pressure inside the device touches the trigger point, a virtual bullet will be generated and shown on the screen, which has the same physical behavior as the real bullets do. This will be explained in Section 4.2. The application will detect the collision of the bullet and the target, calculate the point user get.

We designed the VBS system as a virtual training field now, which uses big-size screens. However, the system can also be used for home or office, by simply connect the VBS device (USB) and USB camera to PC.

\section{Visual Simulation}

\subsection{View-Dependent Virtual Reality Using Face Tracking}

We propose a simple way of increasing the quality of virtual environment visually, using face tracking technologies, which requires no markers or devices binding on user's body. Only a USB camera, set on the top of the display device, and appropriate lightening are required.

We are currently using faceAPI from seeingmachines[8] for 3D face tracking. Both face's position and orientation is tracked real time, in order to measure raw gaze line.

Through our research we found out that instead of moving eyeball, user prefers move head when he is trying to gaze on something during exercise. So we just simply assume that there is no eyeball movement and the normal vector of user's face can be considered as user's gaze line.

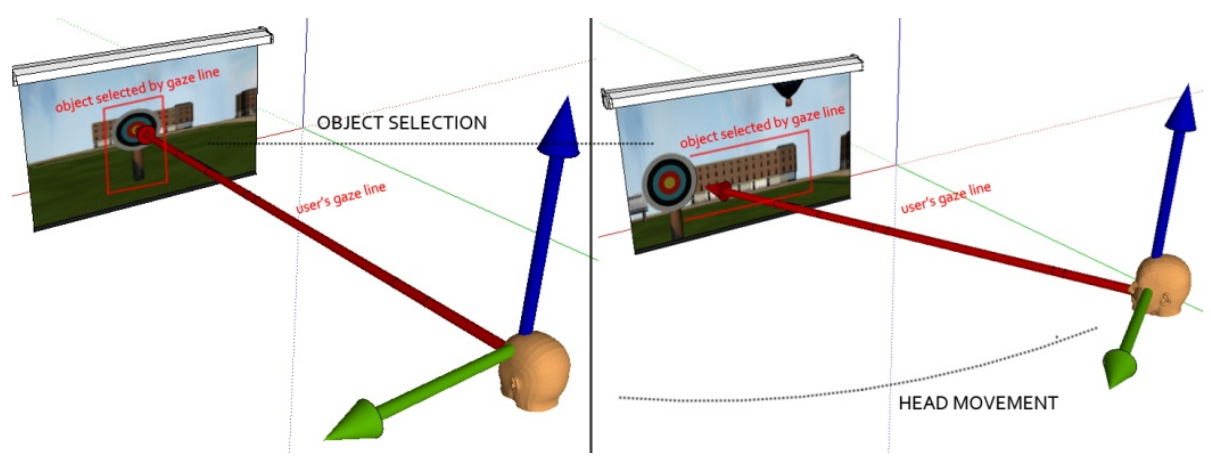

Fig. 4. Synchronize of user's face and virtual camera, and gaze line-base object selection

We suppose user's initial view point is to the center of the display, and user's face is also in the center of the image captured by the camera. When user's head moves, the virtual camera in the application will also moves, synchronized with user's gaze line. (Fig. 4) 
Not only the VR camera's position, but also the position the camera focuses on will be changed. Consider the target is on the center of the screen, when user is trying to aim, or focus his sight on the target, he/she will probably move his/her face above the center point. When user moves his/her face away from center, he/she is probably trying to see something behind the target or something far away.

We consider the effect of visual psychophysics, by using deformation, zoom-in, blurring, etc, to enhance user's visual experience.

User's gaze line is supposed as a beam, shot into the virtual scene generated by the application, and hits the object in the scene. The gaze line can be used in several ways:

- The angle between the gaze line and the object's front plane normal can be calculated so we can create deformation on objects in the VR scene, raise the quality of immersive feelings. [10]

- When user gazing at an object for a period of time, trying to focusing on something (take aim at something for example), objects irrelevant to this action will be blurred.

\subsection{Physical Simulation of Blowgun Bullet}

We integrate the virtual training space into real world. One unit in the virtual space is set as 1/1000 meter. As shown in Fig.5, in real world, distance from user to screen is 3 meters, in virtual space distance from screen to target is 7 meters. Height of target will be set the same as user's height, so different people at different height will not feel uncomfortable when using the system.

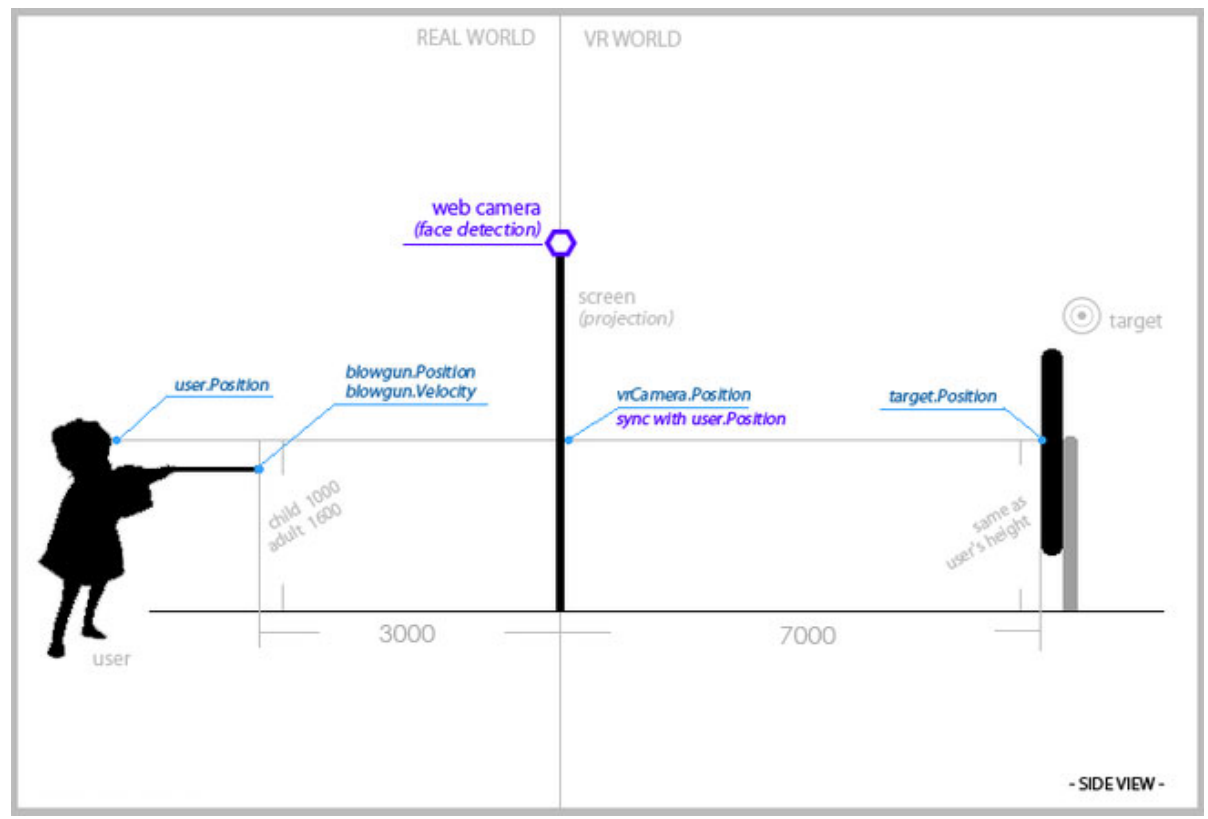

Fig. 5. Integration of virtual world with real world 
We have built a physical model, which can simulate the movement of the bullet, considering the effect of the gravitation and the resistance forces.

The simulation depends on two parameters: the initial velocity vector and the initial XYZ position of the bullet.

Initial velocity vector can be acquired from sensor module. The 3-axis magnetic sensor for YAW-angle, and the 3-axis acceleration sensor for PITCH-angle.

Concerning magnetic field changes due to location or the interference of electronic and magnetic devices like PC, magnetic sensor needs to be initialized, by simply point the VBS device vertically to the display. We also need to know the scalar initial speed of the bullet, which we set to $50 \mathrm{~m} / \mathrm{s}$. This value will be associated with the inner pressure of the device in our future work.

Initial XYZ position of the bullet is considered to be at the same position of user's face.

The application will check the collision between the bullet and the target, and will be marked clearly to tell user whether the bullet is hit the target or not.

\section{Future Works}

Our research will be continued and focused on the following two major themes:

1. Method of creating highly simulated versatile training field, which can also be easily setup at home or office with rare limitations.

2. The application of view-dependent virtual reality with face tracking technologies in versatile training fields.

Currently, the design of the VBS device is still in experimental stage. The following parts of the device will be re-designed or added:

- Automatic pressure control unit.

- Cable-free VBS device. We will use wireless module (bluetooth) instead of USB module for sensors' data transferring.

- The sensor unit will be merged into one narrow board, and as small as possible.

We also have plans of:

- Creating game contents which are able to control user's breathing movement.

- Enable online battles between players.

\section{References}

1. http://www.buteykolearning.com/

2. Cooper, S., Oborne, J., Newton, S., Harrison, V., Thompson Coon, J., Lewis, S., Tattersfield, A.: Effect of two breathing exercises (Buteyko and pranayama) in asthma: a randomised controlled trial. Thorax Aug. 58(8), 674-679 (2003)

3. IJsselsteijn, W., de Kort, Y., Westerink, J., de Jager, M., Bonants, R.: Fun and sports: Enhancing the Home Fitness Experience. In: Rauterberg, M. (ed.) ICEC 2004. LNCS, vol. 3166, pp. 46-56. Springer, Heidelberg (2004) 
4. Mokka, S., Väätänen, A., Heinilä, J., Välkkynen, P.: Fitness Computer Game with a Bodily User Interface. In: Proceeding of the Second International Conference on Entertainment Computing, pp. 1-3 (2003)

5. Tsuji, T., Sumida, Y., Kaneko, M., Sadao: A Virtual Sports System for Skill Training. Journal of Robotics and Mechatronics 13(2), 168-175 (2001)

6. Masuko, S., Hoshino, J.: An Exercise Game Reflecting Heart Rate. The Journal of the Society for Art and Science 6(3), 136-144 (2007) (in Japanese)

7. Yuuichirou, M., Ishiguro, K., Yasuo, O.: Simplified Apparatus for the Abdominal Breathing Exercise. The Journal of Japanese Physical Therapy Association 22(7), 413-416 (1995) (in Japanese)

8. http://www. seeingmachines.com/product/faceapi/

9. Takayuki, N., Terukazu, H., Yokiko, G., Yukiko, M., Hirotaka, O., Hironobu, Y., Hideaki, F., Satoru, K., Seiichiro, T.: Effects of Blowgun Training on Respiatory Functions: For Applied Pulmonary Therapy. The Journal of Kyushu University of Nursing and Social Welfare 9(1), 3-6 (2007) (in Japanese)

10. Owada, S., Fujiki, J.: Ambiguous Rendering for Suppressing Deformation Effect for Stereoscopic Viewing of Spatially Inconsistent Scenes. ITE Technical Report 32(27), 21-24 (2008) (in japenese) 